\title{
A Novel Criterion of Mixing Time in Gas-Stirred Ladle Systems
}

\author{
Shanqiang Ni $\cdot$ Haijuan Wang $\cdot$ Jun Zhang $\cdot$ Lu Lin $\cdot$ Shaojun Chu
}

Received: 16 December 2013/Revised: 26 March 2014/Published online: 1 August 2014

(C) The Chinese Society for Metals and Springer-Verlag Berlin Heidelberg 2014

\begin{abstract}
A novel criterion of mixing time in gas-stirred ladle systems was proposed in this paper. The essential difference between the previous criterion and the present one is that the former is based on the concentration itself while the latter is based on the variation rate of the concentration, which reflects the mixing efficiency directly. Experiment was carried out in an acrylic glass vessel with bottom blowing, and the tracer concentration was monitored by electrical conductivity probes. The mixing time obtained through the new criterion is approximately $20 \%$ less than that obtained through the 95\% criterion, and the deviations of mixing times calculated from the new criterion are smaller than that from the previous one under the same conditions.
\end{abstract}

KEY WORDS: Mixing time; Gas flow rate; Mixing intensity criterion

\section{Introduction}

Mixing plays an important role in metallurgical processes. For the determination of mixing time in stirred vessel by means of conductivity probes, the vessel is filled with water, and a small volume of a tracer material (e.g., a salt solution) is fed into the vessel. The variation of concentration with time is then measured by means of probes. Dackwerts [1] had precisely described the mixing process in his works. When the tracer was injected into the vessel, it was broken up into "clumps", which were intermingled

Available online at http://link.springer.com/journal/40195

S. Ni $\cdot$ H. Wang $(\bowtie) \cdot$ L. Lin $\cdot$ S. Chu

Department of Ferrous Metallurgy, School of Metallurgical and

Ecological Engineering, University of Science and Technology

Beijing, Beijing 100083, China

e-mail: wanghaijuan@ustb.edu.cn

J. Zhang

School of Metallurgy and Resource, Anhui University of

Technology, Ma'anshan 243002, China into the bulk. At the same time, molecular diffusion of the tracer occurred across the boundaries of the clumps. The mechanical stirring broke the tracer into smaller clumps continuously, which sped up the mixing process. In order to define the degree of mixing, Dackwerts [1] proposed the concept of "intensity of segregation" based on statistical theory, which reflected the extent of concentration in the clumps departing from the mean. Krishnamurthy [2] expressed the intensity of segregation of the tracer element in the gas-agitated bath as

$I_{\mathrm{s}}=\left|\left(C_{\infty}-C_{(t)}\right) /\left(C_{\infty}-C_{0}\right)\right|$,

where $C_{\infty}$ and $C_{0}$ refer to the final and initial average uniform concentrations of the tracer, respectively. $C_{(t)}$ is the tracer uniform concentration of any small "clump" of the bath at time $t$. The degree of mixing, $Y$, which represents the extent of mixing in the bath, is defined as

$Y=1-I_{\mathrm{s}}$.

The mixing time $t_{Y}$ denotes the time requirement for reaching the mixing degree $Y$. The determined mixing time is different for different values of $Y$ [3], $Y=0.95$ was employed in most of the previous studies [4-9]. 
Furthermore, the mixing time is also position-dependent [10], hence the mixing time determined in the dead zone is defined as the mixing time of the vessel [11]. This criterion of mixing time is the so-called $95 \%$ criterion. It is widely employed in the recent investigations about influential factors of determined mixing time in gas-stirred ladles such as gas flow rate, gas injection positions, monitoring points, tracer injection positions, and tracer concentration [12-17].

However, there are two drawbacks for the $95 \%$ criterion which are impossible to be ignored: (1) if the dead zone is absolutely inactive and is much smaller compared to the bulk, it will take very long time to get homogenous state of the dead zone other than the whole bulk; under this situation, the mixing time determined in this region could not represent the bulk; (2) Considering the production efficiency, the absolute homogeneity of the bath,which is unnecessary in the industrial process, only leads to the increase of the cost, therefore, the longest mixing time might not be practicable in some cases. For these reasons, the authors proposed a novel criterion to define the mixing time based on the rate of concentration variation of tracer, in order to obtain a better determination of homogenous state of the whole bulk at the same time more practical in production judgement.

\section{Criterion of Mixing Time}

The rate of tracer concentration variation, denoted by " $R$ ", is introduced to describe the mixing intensity at different stage of mixing process, and it can be expressed as

$R=\left|\frac{\mathrm{d} C_{(t)}}{\mathrm{d} t}\right|$,

where $C_{(t)}$ is the concentration varying with time after the tracer injected. However, it is impossible to determine the tracer concentration continuously in practice, and the determined values are always discrete. So Eq. (3) is rewritten as following:

$R=\left|\Delta C_{(t)} / \Delta t\right|$,

where $\Delta t$ is the sampling interval and $\Delta C_{(t)}$ is the concentration difference in this period. It is obvious that the value of $R$ defined here depends on the magnitude of $\Delta t$. In the present experiment, the sampling interval was set to be $1 \mathrm{~s}$. The mean mixing intensity of the bath, which is denoted by $\bar{I}$, is defined as

$$
\begin{aligned}
\bar{I} & =\left(R_{1}+\cdots+R_{n}\right) / n \\
& =\left(\left|\Delta C_{1(t)} / \Delta t\right|+\cdots+\left|\Delta C_{n(t)} / \Delta t\right|\right) / n,
\end{aligned}
$$

where $C_{n(t)}$ is the local concentration at the measured position which was numbered as $n$. The absolute value sign

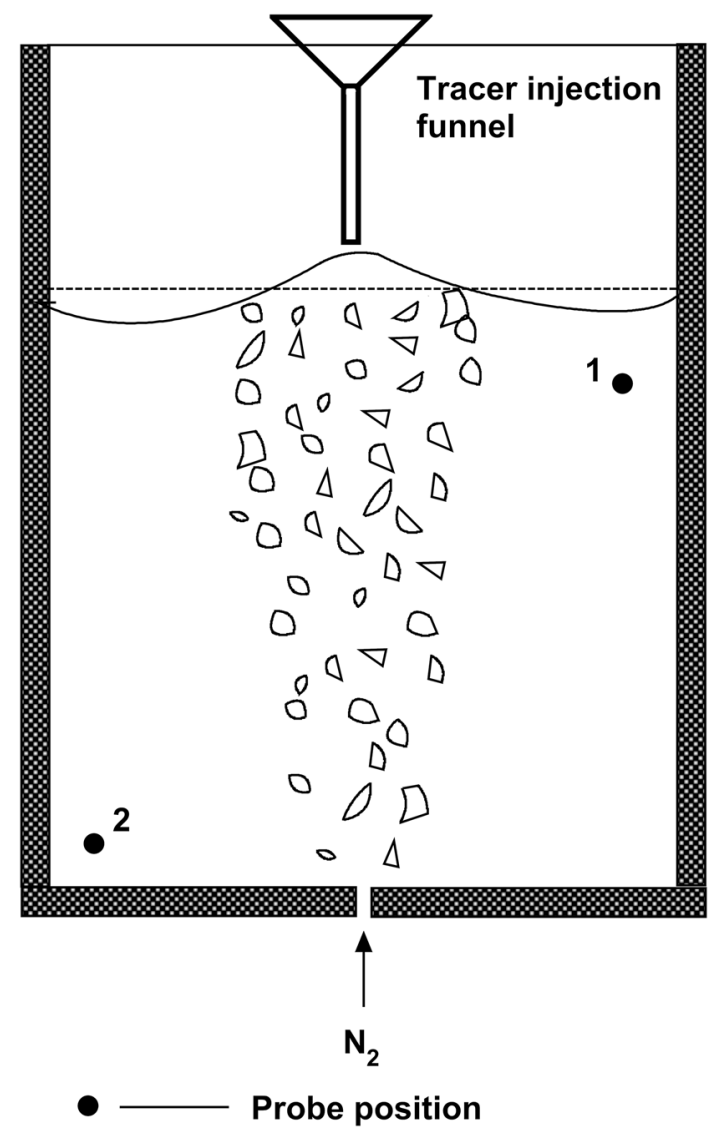

Fig. 1 Schematic of experimental apparatus

here is to avoid the neutralization of the positive and negative values. Theoretically, $\bar{I}=0$ indicates that the tracer concentrations remain constant at all determined positions, and the bath reached the homogeneous state already. However, due to the presence of the instrumental error, the measured tracer concentration value would be always fluctuating in an extent even when the bath is homogeneous. The maximum fluctuation value of $\bar{I}$ in a uniform bath was denoted by $\sigma$. The mixing time was defined as the period starting from the moment of tracer being injected to the moment of $\bar{I}<\sigma$.

\section{Experimental}

Experiments were carried out in a cylindrical vessel of acrylic glass with an inner diameter of $750 \mathrm{~mm}$ and a height of $1,000 \mathrm{~mm}$ at room temperature. The height of water filling the vessel was $900 \mathrm{~mm}$. Water bath was agitated by injecting $\mathrm{N}_{2}$ through a nozzle located axially at the bottom of the vessel. Figure 1 shows the schematic of the experimental apparatus. 


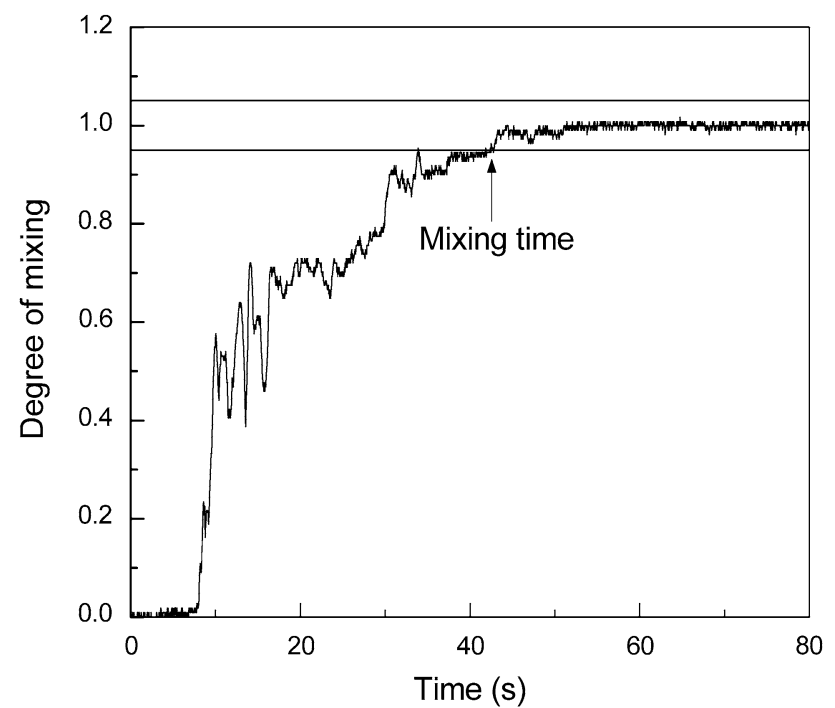

Fig. 2 Definition of mixing time with $95 \%$ criterion

The tracer substance to be mixed was an aqueous $\mathrm{KCl}$ solution with a concentration of $3 \mathrm{~mol} / \mathrm{L}$. $200 \mathrm{~mL}$ of saturated $\mathrm{KCl}$ solution was added directly to a point on the liquid-free surface through a funnel. Two electrical conductivity probes were employed, one located in the active zone (position 1) while the other is in the dead zone (position 2) (Fig. 1). The variation of local tracer concentration around the probe tip was measured through the variation in water's electrical conductivity and recorded in a computer via a digital conductivity meter. For each experiment, the recording of tracer response was carried out until the concentration of the tracer in the bath was considered to have reached a homogeneously mixed value. A set of four measurements were made for each experimental condition.

\section{Comparison of Mixing Times Obtained from Two Criteria}

As mentioned above, with $95 \%$ criterion, the mixing time defined as a period for the tracer concentration in the slowest mixing region has fallen within a $\pm 5 \%$ deviation around the final constant tracer concentration in the bath, as shown in Fig. 2. In present study, the mixing time at position 2 shown in Fig. 1 was selected as the mixing time of the whole bath if $95 \%$ criterion was chosen.

As for the mixing intensity criterion, the value of mean mixing intensity in the present experiment could be expressed as following:

$\bar{I}=\left(\left|\Delta C_{1(t)} / \Delta t\right|+\left|\Delta C_{2(t)} / \Delta t\right|\right) / 2$,

where $C_{1(t)}$ and $C_{2(t)}$ are the tracer concentrations at position 1 and point 2 at time $t$, respectively (refer to

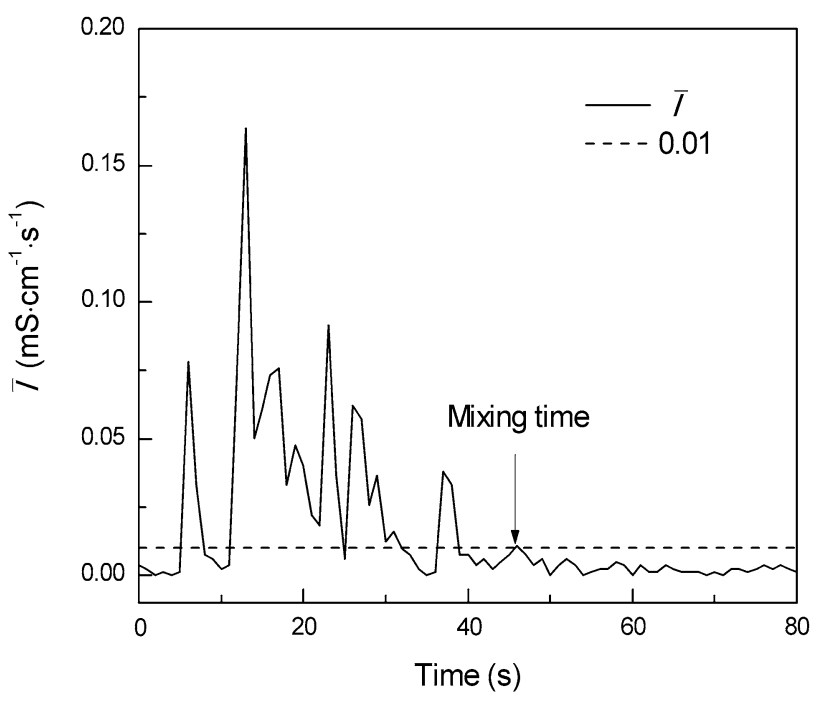

Fig. 3 Definition of mixing time with mixing intensity criterion

Fig. 1).The maximum of mean mixing intensity $\sigma$ in a uniform bath was measured as $0.01 \mathrm{mScm}^{-1} \mathrm{~s}^{-1}$ for the present experiment. Therefore, the authors believe as long as the fluctuation of $\bar{I}$ is within $0.01 \mathrm{mScm}^{-1} \mathrm{~s}^{-1}$, the solution is in homogeneous state. A typical example for definition of mixing time with mixing intensity criterion is shown in Fig. 3. The curve fluctuates greatly from the onset of the tracer injection and is below $0.01 \mathrm{mScm}^{-1} \mathrm{~s}^{-1}$ after $46 \mathrm{~s}$, which indicates that the mixing time is $46 \mathrm{~s}$ in this situation.

The mixing times were calculated with two different criteria through the same measured data set. The essential difference of the $95 \%$ criterion and the present one is that the former is based on the concentration itself while the latter is based on the variation rate of the concentration, which reflects the mixing efficiency directly. In addition, for the $95 \%$ criterion, only the longest mixing time determined in the dead zone was taken as the mixing time of the bath, while for the mixing intensity criterion, both of the dead zone and the active zone were considered, which is more reasonable.

Figure 4 shows a comparison of the mixing times determined with the mixing intensity criterion and the $95 \%$ criterion. For both criteria, the mixing times considerably decrease with the increasing volume flow rate of the stirring gas. However, it can be seen that the former is approximately $20 \%$ less than the latter under the same experimental conditions, just because the different criterion was applied. According to the present study, the mixing intensity in the bath is relatively weak after the homogeneity was obtained based on the new criterion, even though the tracer concentration doesn't fall continuously within a $5 \%$ deviation of the well homogeneous value. Therefore, 


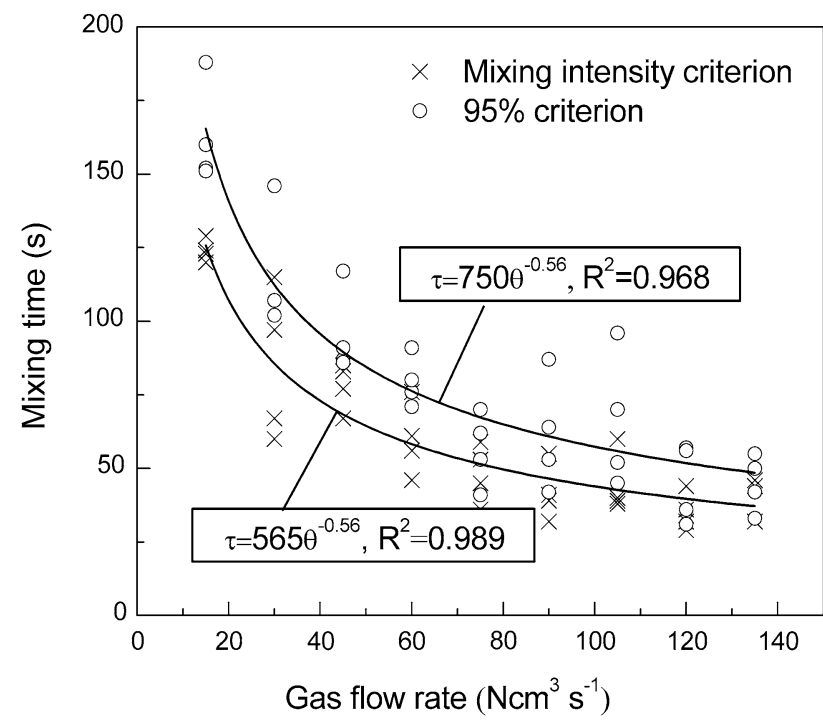

Fig. 4 Comparison of mixing time values with two criteria

the time difference between mixing time measured by the current criterion and the $95 \%$ criterion could be taken as "extra time", and only a weak gas blowing was needed to protect the porous plug from erosion in that stage, which is good for saving energy and increasing efficiency in the industry.

It is also shown in Fig. 4 that the deviations of mixing times calculated from the new criterion are smaller than that from the previous one under the same conditions. It is useful for the analysis of the experimental data.

The curves drawn in Fig. 4 were obtained by regression analysis of the measured values according to a function presented in the following equation:

$t_{\text {mix }}=a Q^{b}$,

where $a, b$ are the constants obtained from regression analysis and $Q$ is the volume flow rate of stirring gas. To avoid the dimensional inhomogeneity, it is assumed that $t_{0}=1 \mathrm{~s}$ and $Q_{0}=1 \mathrm{Ncm}^{3} / \mathrm{s}$. So, the empirical equations could be expressed by the dimensional time $\tau=t_{\text {mix }} / t_{0}$ and the dimensional gas flow rate $\theta=Q / Q_{0}$.

\section{Conclusions}

(1) The mixing time values obtained through the new criterion were approximately $20 \%$ less than that through the $95 \%$ criterion. Since only a weak gas blowing was needed to protect the porous plug from erosion after the homogenous stage in the industrial process, the new criterion could be good for saving energy and increasing efficiency in the industry.

(2) The deviations of mixing time for different experiments under new criterion are smaller than that with the previous criterion at the same gas flow rate.

Acknowledgments This work was financially supported by the National Natural Science Foundation of China (Nos. 51274030 and 51204001).

\section{References}

[1] P.V. Danckwarts, Appl. Sci. Res. A 3, 279 (1952)

[2] G.G. Krishnamurthy, J.F. Elliott, ISIJ Int. 32, 190 (1992)

[3] G.G. Krishnamurthy, ISIJ Int. 29, 49 (1989)

[4] D. Mazumdar, R.I.L. Guthrie, Ironmak. Steelmak. 12, 256 (1985)

[5] Y. Takatsuka, M. Iguchi, ISIJ Int. 41, 124 (2001)

[6] P. Ternstedt, A. Tilliander, P.G. Jonsson, M. Iguchi, ISIJ Int. 50, 663 (2010)

[7] D.Q. Geng, H. Lei, J.C. He, Int. J. Min. Metall. Mater 17, 709 (2010)

[8] H.J. Odenthal, U. Thiedemann, U. Falkenreck, J. Schlueter, Metall. Mater. Trans. B 41, 396 (2010)

[9] A.N. Conejo, S. Kitamura, N. Maruoka, S.J. Kim, Metall. Mater. Trans. B 44, 914 (2013)

[10] D. Mazumdar, R.I.L. Guthrie, ISIJ Int. 35, 220 (1995)

[11] D. Mazumdar, D. Santanu, B. Sudhendu, ISIJ Int. 37, 194 (1997)

[12] L.T. Khajavi, M. Barati, Metall. Mater. Trans. B 41, 86 (2010)

[13] X.G. Ai, Y.P. Bao, W. Jiang, Int. J. Min. Metall. Mater. 17, 17 (2010)

[14] D. Mazumdar, R.I.L. Guthrie, Metall. Mater. Trans. B 41, 976 (2010)

[15] N. Kochi, K. Mori, Y. Sasaki, M. Iguchi, ISIJ Int. 51, 1755 (2011)

[16] S.P. Patil, D. Satish, M. Peranandhanathan, D. Mazumdar, ISIJ Int. 50, 1117 (2010)

[17] C. Chen, Q. Rui, G. Cheng, Steel Res. Int. 84, 900 (2013) 